\title{
Total revision of the hip using allograft to correct particle disease induced osteolysis: A case study
}

\author{
Drew W. Taylor*, Jennifer E. Taylor, Igal Raizman, Allan E. Gross
}

\begin{abstract}
Total hip replacement is considered to be a highly successful and routine surgery; however, the internal components produce particles through friction and wear in the device. These particles are identified as one of the main reasons for total hip revisions. The generated, biologically active, particles provoke the formation of osteolytic areas through the inhibition of bone formation and increased fluid production. The resulting bone loss can be managed through the use of allograft bone in combination with bone chips and cement. In addition, implants constructed with highly porous trabecular metal can be used to further facilitate rapid and extensive tissue infiltration resulting in strong implant attachment. In this case study we show the use of a tibial allograft coupled with bone chips and cement to cover and support a lytic cyst in the proximal femur, distal to the greater trochanter. Additionally, we detail the use of a trabecular metal cup to halt the migration of the component into the acetabulum and promote greater fixation and bone ingrowth.
\end{abstract}

KEYWORDS: Revision Hip Arthroplasty, Allograft, Osteolysis, Particle Disease, Trabecular Metal

\section{INTRODUCTION}

Total hip replacement is considered to be a highly successful and routine surgery. It is estimated that over a million such medical procedures occur annually world-wide. However, despite the minimal rate of early complications, up to $30 \%$ of all surgeries are revised within 10-14 years of initial surgery (1). Every prosthetic hip replacement available produces particles. These particles are identified as one of the main causal reasons for total hip arthroplasty. The wear between primary binding surfaces of the femoral head and acetabular components in total hip replacements is considered to be the most significant source of prosthetic particles (2).

One estimation, from a study utilizing a metal on polyethylene joint, suggested the generation of hundreds of thousands of polyethylene particles during each gait cycle (3). Another study found that metal-onmetal or ceramic-on-ceramic pairings had significantly

*To whom correspondence should be addressed:

Drew W. Taylor

Mount Sinai Hospital

Division of Orthopaedics

600 University Ave, Room 476A

Toronto, ON (Canada), M5G 1X5

E-mail: drew.w.taylor@gmail.com less wear on the prosthesis (4). However, despite the variances in particle generation of different materials, osteolysis is indicated in all types of hip replacements. Strictly defined, osteolysis refers to an aggressive local bone resorbtion. Particle debris generated from the mechanical wear of prosthetic devices is known to alter the function of multiple cell types in the periprosthetic area, including macrophages, fibroblasts, osteoblasts, and osteoclasts (5-7). The generated biologically active particles appear to provoke the formation of lytic areas through the inhibition of bone formation and increased fluid production (8-10). These cysts ultimately lead to implant loosening.

Bones are highly dynamic tissues undergoing a constant remodeling process that is regulated by a tightly-controlled balance of osteoblast and osteoclast activity. The major challenge of revision hip arthroplasty is related to osteolysis and bone loss decreasing stability of the implant. Revision of the acetabular component can be especially challenging because of deficient bone stock. The loss of bone can surmount due to surgical bone loss from the primary operation, migration of the cup, and as mentioned previously, particle induced osteolysis (11). Consequently, the goal of revision hip surgery should be anatomical placement of the acetabular component, but 
this can be difficult to achieve in cases where contact between host bone and the implant is diminished. This bone loss can be managed by either filling the absent area with cement, the use of allograft bone, or by using special implants such as those constructed with trabecular metal (12-16). Allograft incorporation can be implemented to reinforce the lytic area, thereby stabilizing the implant. Allografts do not suffer from osteoclastic invasion and it has been shown that osteoblasts deposit osteoid across the host bone and the dead graft (17). This union results in an increased mechanical resistance that is indicated by increased radiological density throughout the graft (18).

The structure of trabecular metal resembles bone and approximates its physical and mechanical properties more closely than other prosthetic materials. The highly porous trabecular configuration is conducive to bone ingrowth, facilitating rapid and extensive tissue infiltration resulting in strong implant attachment (19). Trabecular metal consists of interconnecting pores resulting in a crystalline micro-textured biomaterial that is $80 \%$ porous. This allows for greater tissue ingrowth (2-3 fold higher compared to conventional porous coatings) with approximately double the interface shear strength (19). In hip arthroplasties, porous trabecular metal facilitates bone ingrowth and fixation of the device; multiple studies have shown that most replacements can be reconstructed with an uncemented trabecular hemispherical cup with screws with or without morselized bone graft (20-22). Because of their porous surface and mechanical properties trabecular metal cups provide a promising approach for offering a better environment for bone graft remodeling (23). In this case study we detail the use of a trabecular metal cup to halt the migration of the component into the acetabulum and promote greater fixation and bone ingrowth. Additionally, we show the use of a tibial allograft coupled with bone chips and cement to cover and support a lytic cyst in the proximal femur, distal to the greater trochanter.

\section{CASE STUDY}

A 73 year old man presented with instability in the left hip, 17 years after bilateral hip arthroplasty was completed. The original replacement was a ceramic head on a polyethylene lined acetabular component. Still working full time and an active individual, instability and loss of control began to impede on his daily activities. Sharp pain presented down the lateral aspect of his left leg during jarring actions. During physical examination, good range of motion and gait was displayed in both hips, although pain was produced during forced external rotation of the left hip. Leg length was equal.
$\mathrm{X}$-rays showed significant osteolysis of the proximal femur, distal to the greater trochanter of his left hip (Figure 1). Despite the bone loss, the stem appeared to still be solid. In addition, significant wear of the left cup was observed. It was anticipated that the progressive wear of the polyethylene cup would induce small particle disease involving the greater and lesser trochanter areas. X-rays had been taken 5 years prior to evaluate the aging arthroplasty. Comparison to the current x-rays (Figure 1) revealed that the particle disease had expanded and significantly increased the endosteal lytic zone, it was also evident that the ceramic head had penetrated deeper into the acetabulum. The right hip was not radiologically or symptomatically threatened at the presented time. To avoid impending femoral fracture or avulsion of the greater trochanter from lytic bone loss, revision of the left hip was scheduled.

\section{OPERATIVE TECHNIQUE}

Since the stem had good fixation, the choice was made to leave it in place and use a bone graft to support the lucent area distal to the greater trochanter. A tibial allograft was used to secure the femoral component and stabilize the lytic area. The allograft was irradiated with 2.5 Mrad (25,000 Gy) in the hospital bone bank, accrediated by the American Association of Tissue Banks, and consequently stored at -70 degrees Celsius. The approximate allograft size was templated preoperatively and a longer graft ordered to account for any intraoperative adjustments. The allograft was only brought into the operating room after possible infection of the to-be-revised hip was ruled out. In order to reduce operative time, the allograft was prepared on a separate table by members of the surgical team while the

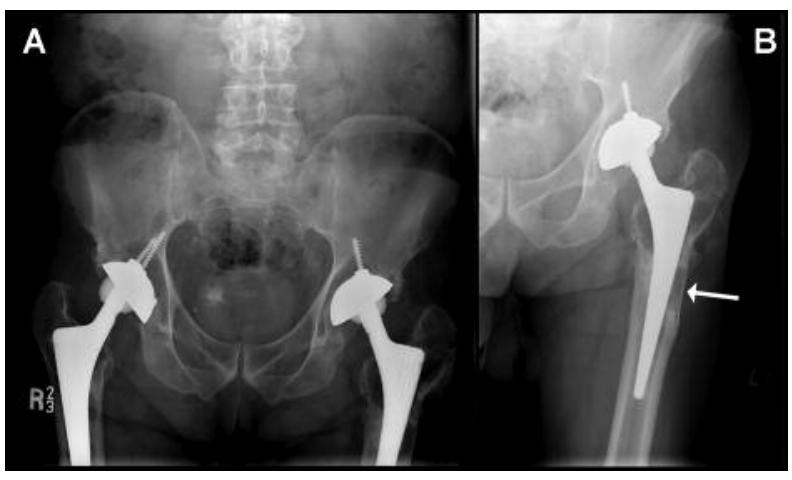

Figure 1: Pre-operative X-rays in the coronal plane. Lucency in the lesser and greater trochanter is evident with the endosteal lytic area marked by a white arrow. It is also apparent that the left acetabular component has pushed deeper into the acetabulum. 
revision was initiated. The femoral component had good fixation so additional bone ingrowth was not a major concern. Thus, cement was used to fill in the lytic area and provide additional structural support. Polymethylmethacrylate bone cement has been known to strengthen allograft bone, impair resorbtion, and allow for the delivery of antibiotics (24). Cement was not used between the allograft and the host bone, only in the lytic area between the allograft and the implant, so as not to impair healing at the host-donor interface. Additionally, morselized bone from the allograft was used between contact sites of host and donor bone in an effort to increase bone ingrowth. Finally wire was used to secure the allograft to the femur.

Operative visualization confirmed the cup had protruded into the acetabulum and wear on the polyethylene lining was evident, necessitating its replacement. A Zimmer Trilogy ${ }^{\mathrm{TM}}$ Acetabular System was selected to replace the acetabular component, consisting of a metal shell, polyethylene liner, and screws. The shell, made from Tivanium ${ }^{\mathrm{TM}}$ Ti-6Al-4V alloy, is pourous to allow for fixation with Tivanium ${ }^{\mathrm{TM}}$ alloy cortical screws. In this case, three $6.5 \mathrm{~mm}$ screws of lengths 25,30 , and $35 \mathrm{~mm}$ were used to secure the cup into the acetabulum. The original cup was uncemented, $56 \mathrm{~mm}$ in size and was replaced with a similar $56 \mathrm{~mm}$ trabecular metal cup. The porous trabecular metal serves to facilitate bone ingrowth and increased fixation of the arthrography. Bone chips from the excess allograft were also used to fill in the protruded acetabulum from the original implant. This will further stimulate bone growth in and around the new trabecular component.

\section{DISCUSSION}

Immediately following the surgery the patient was taken to radiology for post-operative x-rays (Figure 2). Revision of the acetabular component with a trabecular metal cup was then visualized and correct placement

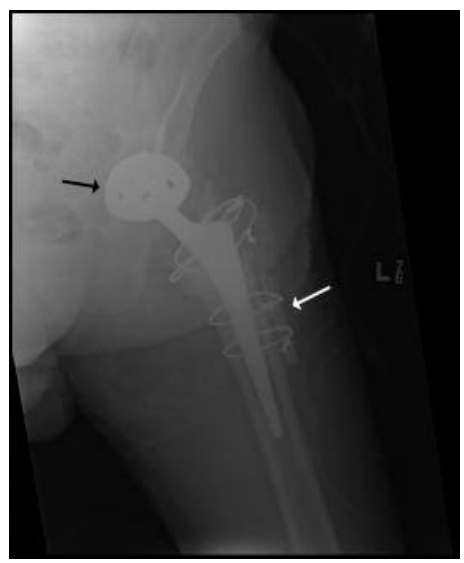

Figure 2: X-ray of the left hip after surgery. Revision of the acetabular component with a trabecular metal cup is marked with a black arrow. The original femoral component is shown reinforced with a tibial allograft, marked by a white arrow. within the acetabulum was confirmed. The original femoral component was also visualized, shown reinforced with a well placed tibial allograft.

Post-operative management of the revision hip replacement includes a diligent rehabilitation program. Revision cases are usually rehabilitated more conservatively than primary replacements, as was the case here. This patient was treated with additional care due to the use of allograft bone to stabilize the femoral component. During the first week post-op, progressive ambulation remains the primary goal. Our patient was encouraged to begin range-of-motion ankle exercises and moving with a walker/assistance 5 to 10 feet the first day after surgery which was done with success. Throughout the first week, aided walking was extended to 25-45 feet and stair-climbing with crutches was introduced after day 5. Stair climbing and other motions that bend the knee must be watched carefully as for the first 3 months the patient is advised not to bend their knee past 90 degrees. This helps to avoid dislocation and damage to the hip and device. The patient progressed very well through the rehabilitation program as proposed.

Additional x-rays were taken after 3 months to confirm fixation of the aetabular component and evaluate the success of the tibial allograft over the osteolytic area in the proximal femur (Figure 3). The acetabular component appeared to be well placed and the observed migration into the acetabulum was halted. The tibial allograft, marked with a white arrow, remains in place supporting the area of osteolysis. The level of

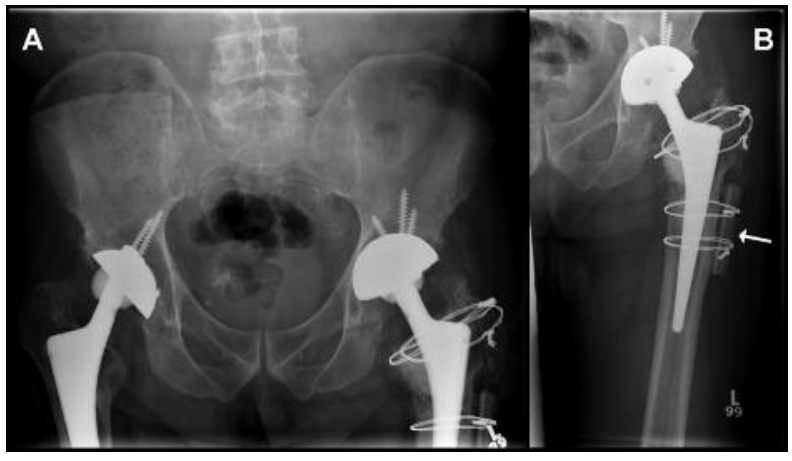

Figure 3: Coronal X-rays taken 3 months post-operatively. The acetabular component appears to be well placed and the progressive push into the acetabulum appears to be corrected. The tibial allograft, marked with a white arrow, remains in place supporting the area of osteolysis. The level of transparency has reduced significantly after cleaning out the cyst and filling the lytic zone with biocompatible cement. 
transparency had reduced significantly after successful cleaning of the cyst and filling the lytic zone with biocompatible polymethylmethacrylate bone cement. Physical examination was completed at the same time as X-rays to ensure the patient had continued with the rehabilitation program and was continuing to make significant progress. Ambulation, range of motion, and functionality were all observed and were progressing well. The patient is now fully recovered, ambulating and functioning without pain or discomfort.

For reasons including particle disease, revision of total hip replacements generally occur upon indication of a painful loose prosthesis. Davis et al. (25) used the Western Ontario and McMaster Universities Osteoarthritis (WOMAC) questionnaire to grade pain and function pre- and 2-year-postoperatively. The study reported that a higher pain level and number of comorbidities before the surgery predicted poorer outcomes at 24 month post-surgical follow-up. Patients with better preoperative WOMAC pain and function scores had better scores postoperatively, suggesting the benefit of performing total hip revision not only to relieve current symptoms, but to reduce the chance that pain and function will worsen while waiting for surgery. In this case, loosening was first noticed by the patient through instability and loss of gait control and later confirmed through radiography. If surgery had waited until higher levels of pain were expressed to maximize the life of the original device, more serious complications may have occurred. By performing the revision more complicated surgeries with worse outcomes, potentially for failure due to fracture, are avoided. The use of allograft is shown as an effective way to support components threatened with osteolysis. Donor bone in combination with biocompatible cement can greatly reduce the potential for injury and pain due to lytic bone loss.

\section{REFERENCES}

1. Duffy, G. P., Berry, D. J., Rowland, C., and Cabanela, M. E. Primary uncemented total hip arthroplasty in patients $<40$ years old: 10- to 14-year results using first-generation proximally porous-coated implants. J Arthroplasty. 2001;16:140-144.

2. Schmalzried, T. P. and Callaghan, J. J. Wear in total hip and knee replacements. J Bone Joint Surg Am. 1999;81:115-136.

3. McKellop, H. A., Campbell, P., Park, S. H., Schmalzried, T. P., Grigoris, P., Amstutz, H. C., and Sarmiento, A. The origin of submicron polyethylene wear debris in total hip arthroplasty. Clin Orthop Relat Res. 1995;3-20.

4. Ingham, E. and Fisher, J. Biological reactions to wear debris in total joint replacement. Proc Inst Mech Eng [H ]. 2000;214:2137.

5. Gallo, J., Kaminek, P., Ticha, V., Rihakova, P., and Ditmar, R. Particle disease. A comprehensive theory of periprosthetic osteolysis: a review. Biomed Pap Med Fac Univ Palacky Olomouc Czech Repub. 2002;146:21-28.

6. Nakashima, Y., Sun, D. H., Trindade, M. C., Maloney, W. J., Goodman, S. B., Schurman, D. J., and Smith, R. L. Signaling pathways for tumor necrosis factor-alpha and interleukin-6 expression in human macrophages exposed to titanium-alloy particulate debris in vitro. J Bone Joint Surg Am. 1999;81:603615.

7. Vermes, C., Chandrasekaran, R., Jacobs, J. J., Galante, J. O., Roebuck, K. A., and Glant, T. T. The effects of particulate wear debris, cytokines, and growth factors on the functions of MG-63 osteoblasts. J Bone Joint Surg Am. 2001;83-A:201-211.

8. Willert, H. G., Buchhorn, G. H., and Hess, T. [The significance of wear and material fatigue in loosening of hip prostheses]. Orthopade. 1989;18:350-369.

9. Willert, H. G., Bertram, H., and Buchhorn, G. H. Osteolysis in alloarthroplasty of the hip. The role of ultra-high molecular weight polyethylene wear particles. Clin Orthop Relat Res. 1990;95-107.

10. Willert, H. G., Buchhorn, G. H., Gobel, D., Koster, G., Schaffner, S., Schenk, R., and Semlitsch, M. Wear behavior and histopathology of classic cemented metal on metal hip endoprostheses. Clin Orthop Relat Res. 1996;S160-S186.

11. Morag, G., Zalzal, P., Liberman, B., Safir, O., Flint, M., and Gross, A. E. Outcome of revision hip arthroplasty in patients with a previous total hip replacement for developmental dysplasia of the hip. J Bone Joint Surg Br. 2005;87:1068-1072.

12. Gross, A. E., Garbuz, D., and Morsi, E. S. Acetabular allografts for restoration of bone stock in revision arthroplasty of the hip. Instr Course Lect. 1996;45:135-142.

13. Perka, C., Schneider, F., and Labs, K. Revision acetabular arthroplasty using a pedestal cup in patients with previous congenital dislocation of the hip - four case reports and review of treatment. Arch Orthop Trauma Surg. 2002;122:237-240.

14. Shinar, A. A. and Harris, W. H. Bulk structural autogenous grafts and allografts for reconstruction of the acetabulum in total hip arthroplasty. Sixteen-year-average follow-up. J Bone Joint Surg Am. 1997;79:159-168.

15. Tanzer, M. Role and results of the high hip center. Orthop Clin North Am. 1998;29:241-247.

16. Woodgate, I. G., Saleh, K. J., Jaroszynski, G., Agnidis, Z., Woodgate, M. M., and Gross, A. E. Minor column structural acetabular allografts in revision hip arthroplasty. Clin Orthop Relat Res. 2000;75-85.

17. Burchardt, H. The biology of bone graft repair. Clin Orthop Relat Res. 1983;28-42.

18. Gie, G. A., Linder, L., Ling, R. S., Simon, J. P., Slooff, T. J., and Timperley, A. J. Contained morselized allograft in revision total hip arthroplasty. Surgical technique. Orthop Clin North Am. 1993;24:717-725.

19. Bobyn, J. D., Stackpool, G. J., Hacking, S. A., Tanzer, M., and Krygier, J. J. Characteristics of bone ingrowth and interface mechanics of a new porous tantalum biomaterial. J Bone Joint Surg Br. 1999;81:907-914.

20. Engh, C. A., Glassman, A. H., Griffin, W. L., and Mayer, J. G. Results of cementless revision for failed cemented total hip arthroplasty. Clin Orthop Relat Res. 1988;91-110.

21. Harris, W. H. Results of uncemented cups: a critical appraisal at 15 years. Clin Orthop Relat Res. 2003;121-125.

22. la Valle, C. J., Shuaipaj, T., Berger, R. A., Rosenberg, A. G., Shott, S., Jacobs, J. J., and Galante, J. O. Revision of the acetabular component without cement after total hip arthroplasty. A concise follow-up, at fifteen to nineteen years, of a previous report. J Bone Joint Surg Am. 2005;87:1795-1800.

23. Boscainos, P. J., Kellett, C. F., Maury, A. C., Backstein, D., and Gross, A. E. Management of periacetabular bone loss in revision hip arthroplasty. Clin Orthop Relat Res. 2007;465:159-165.

24. Gross, A. E. The role of polymethylmethacrylate bone cement in revision arthroplasty of the hip. Orthop Clin North Am. 2005;36:49-54, vi. 
25. Davis, A. M., Agnidis, Z., Badley, E., Kiss, A., Waddell, J. P., and Gross, A. E. Predictors of functional outcome two years following revision hip arthroplasty. J Bone Joint Surg Am. 2006;88:685-691.

Drew W. Taylor (M.S., PhD (2010)) is currently in a PhD in Biomedical Engineering at the University of Toronto. He received his B.S. and M.S. in Molecular, Cellular, and Developmental Biology from the University of Michigan.

Jennifer E. Taylor (M.S. (2010), B.A., CPT) is currently working in Strength and Conditioning in the Athletic Department at the University of Michigan while completing her Masters in Exercise Physiology. She completed her Bachelor's degree in Kinesiology at the University of Michigan.

Igal Raizman (B.S., M.S. (2010)) is currently in a Masters program in Laboratory Medicine and Pathobiology at the University of Toronto. He received his Bachelor's degree in Biology and Pharmacology from McMaster University.

Allan E. Gross (M.D., FRCSC, O.Ont.) is an Orthopaedic Surgeon at Mount Sinai Hospital specializing in lower extremity reconstruction, with a particular interest in revision arthroplasty of the hip and knee. He holds the Bernard Ghert Chair in Lower Extremity Reconstructive Surgery and also serves as a professor in the Department of Surgery at the University of Toronto. 